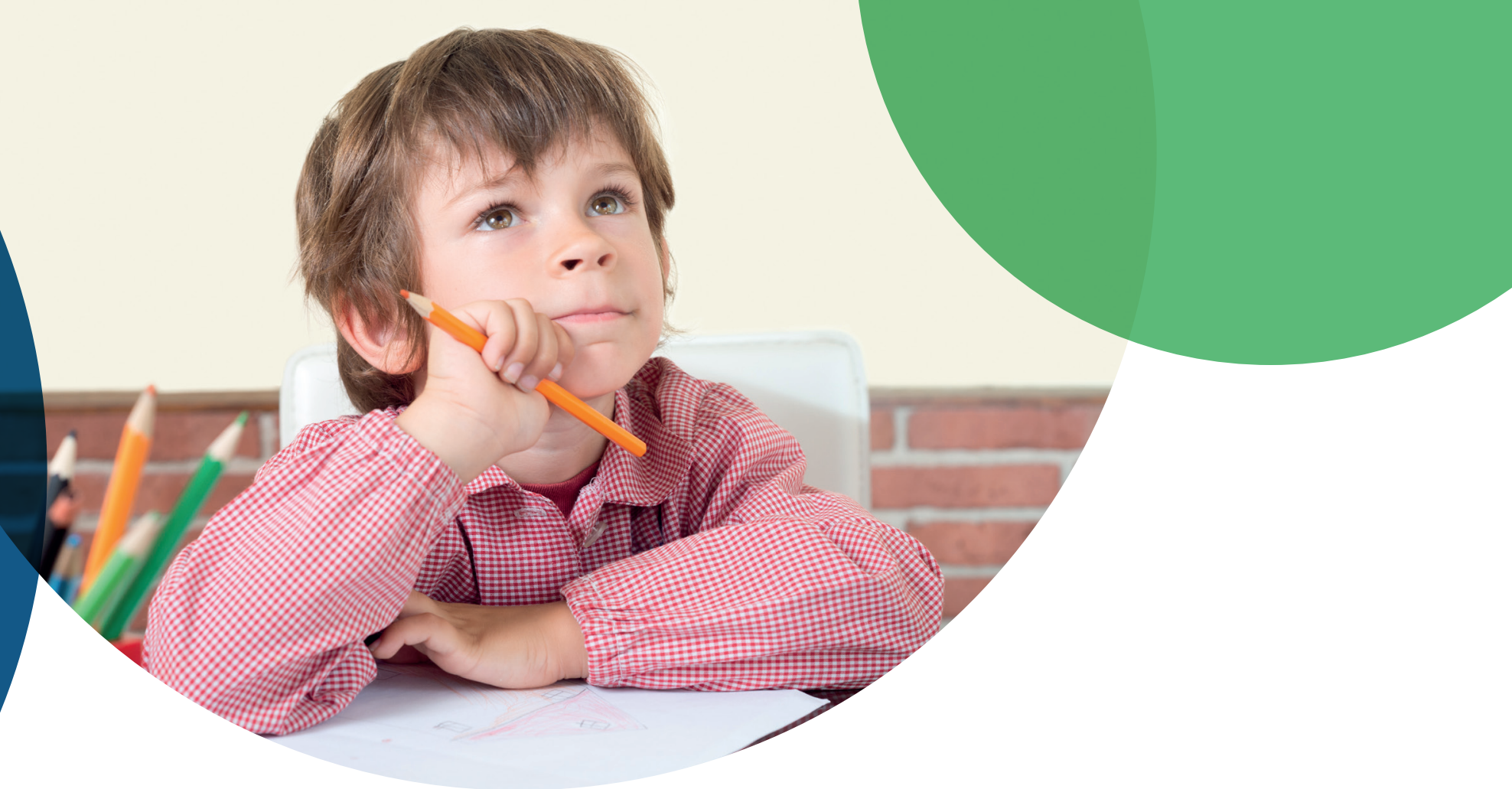

\title{
Brain firing, but not wiring, is different in children with ADHD
}

\author{
When in a relaxed state, the brains of children and adolescents with ADHD tend to fire differently \\ to those without the disorder, although there don't seem to be changes in the physical connections \\ or 'wiring' of their brains.
}

ADHD symptoms are thought to be underpinned by changes in brain connectivity, but the details of these remain uncertain. To address this, researchers in the Netherlands based in Utrecht and Leiden used MRI brain scanning to examine two forms of brain connectivity.

They investigated both the structural connectivity - the physical connections between brain areas, and functional connectivity - seeing if brain areas fire at the same time and are therefore likely to be communicating.

Previous behavioural and brain scanning studies have found some evidence that the brains of those with ADHD mature differently - this has led to the development of the 'maturational delay hypothesis' of ADHD.

"Brain development literally lags behind a few years," said Dr Dienke Bos of University Medical Centre Utrecht, one of the authors of the study. She said other evidence has shown a delay in those with ADHD of two to five years in the frontal cortex - a brain region associated with abstract thought, decision-making and short term memory.

But the situation is complex; the hypothesis doesn't explain why some children's ADHD continues into adulthood.

"In many children this delay seems to go away after adolescence" she said.

"The maturational delay hypothesis is one of the prevailing hypotheses of brain development in ADHD," she said "It's not perfect, but it's the best we have at the moment."
The researchers focussed their investigations into the firing patterns of a well-established grouping of brain areas that tend to become active when we are resting our attention, known as the default mode network (DMN).

"The DMN is something we should only see when you're doing nothing," said Dienke.

"In children with ADHD we see it pop up when we ask them to do something, which is probably associated with inattention," she said.

The researchers scanned the brains of 35 children and adolescents with ADHD and 36 without. They used both functional magnetic resonance imaging (fMRI) - which measures brain activity through blood flow, and diffusionweighted imaging (DWI) - which measures brain cell connections through the flow of water.

The researchers found differences in the functional connectivity of the ADHD children, but not in their structural connectivity.

"That was definitely surprising, as we did find some fairly clear functional differences," said Dienke.

She reflected that scanning the brains of children is not without its problems, particularly if they have ADHD.

"It's really tough," said Dienke. "I think we lost about thirty to forty percent of our data in the resting state study," she said, speaking about the functional brain scans. 
The researchers used a movie to distract the children during the structural scans, but they had to rest their attention for the functional scans, meaning they could only stare at a white cross on a black background.

"Right now we have to include a lot of children and hope for the best," she said.

Having a brain that's slow to grow up isn't enough to explain all aspects of ADHD. "It's not the full story," said Dienke. "There are functional and structural changes that appear throughout development" she said.

The researchers suggest such functional differences, which may be associated with the disruption of brain networks, could come before more permanent changes in wiring.

Further work will be required to make a direct link between the functional and structural changes.

"One of the interesting things for us would be to follow up on these children, and see if there are still no differences, or if differences appear later," said Dienke.

This kind of follow up research - known as a longitudinal study - could also explore possible links between changes in brain function and structure and ADHD symptom severity.

"We actually have longitudinal data that we're analysing right now," said Dienke.

Hopefully further work looking at the brains of children with ADHD as they grow up will teach us more about the subtle changes that underlie this common, but varied, disorder.

Bos, D. J., Oranje, B., Achterberg, M., Vlaskamp, C., Ambrosino, S., de Reus, M. A., van den Heuvel, M. P., Rombouts, S. A.R.B. and Durston, S. (2017), Structural and functional connectivity in children and adolescents with and without attention deficit/ hyperactivity disorder. J Child Psychol Psychiatr, 58: 810-818. doi:10.1111/jcpp.12712

Default mode network: A specific group of brain regions, which are usually active when an individual is resting, or not completing any sort of task.

Structural connectivity: The amount of physical connections a brain area has with other areas. These are also known as white matter, or axons, and carry electrical signals.

Functional connectivity: The amount two brain areas actually communicate, based on the fact they are active at the same time, such synchronised firing generally results in stronger physical connections over time. 